\title{
BMJ Open Using mixed methods to assess fidelity of delivery and its influencing factors in a complex self-management intervention for people with osteoarthritis and low back pain
}

\author{
Elaine Toomey, ${ }^{1}$ James Matthews, ${ }^{2}$ Deirdre A Hurley ${ }^{2}$
}

To cite: Toomey E, Matthews J, Hurley DA. Using mixed methods to assess fidelity of delivery and its influencing factors in a complex selfmanagement intervention for people with osteoarthritis and low back pain. BMJ Open 2017;7:e015452. doi:10.1136/ bmjopen-2016-015452

- Prepublication history and additional material for this paper are available online. To view these files please visit the journal online (http://dx.doi. org/10.1136/bmjopen-2016015452).

Received 5 December 2016 Revised 31 March 2017 Accepted 5 May 2017

\section{CrossMark}

${ }^{1}$ School of Psychology, Arts Millennium Building, National University of Ireland, Galway, Ireland

${ }^{2}$ School of Public Health, Physiotherapy and Sports Science, Health Sciences Centre, University College Dublin, Belfield, Ireland

Correspondence to

Dr Elaine Toomey;

elaine.toomey@nuigalway.ie

\section{ABSTRACT}

Objectives and design Despite an increasing awareness of the importance of fidelity of delivery within complex behaviour change interventions, it is often poorly assessed. This mixed methods study aimed to establish the fidelity of delivery of a complex self-management intervention and explore the reasons for these findings using a convergent/ triangulation design.

Setting Feasibility trial of the Self-management of Osteoarthritis and Low back pain through Activity and Skills (SOLAS) intervention (ISRCTN49875385), delivered in primary care physiotherapy.

Methods and outcomes 60 SOLAS sessions were delivered across seven sites by nine physiotherapists. Fidelity of delivery of prespecified intervention components was evaluated using (1) audio-recordings ( $n=60)$, direct observations $(n=24)$ and self-report checklists $(n=60)$ and (2) individual interviews with physiotherapists $(n=9)$. Quantitatively, fidelity scores were calculated using percentage means and SD of components delivered. Associations between fidelity scores and physiotherapist variables were analysed using Spearman's correlations. Interviews were analysed using thematic analysis to explore potential reasons for fidelity scores. Integration of quantitative and qualitative data occurred at an interpretation level using triangulation.

Results Quantitatively, fidelity scores were high for all assessment methods; with self-report $(92.7 \%)$ consistently higher than direct observations $(82.7 \%$ ) or audio-recordings (81.7\%). There was significant variation between physiotherapists' individual scores $(69.8 \%$ - 100\%). Both qualitative and quantitative data (from physiotherapist variables) found that physiotherapists' knowledge (Spearman's association at $p=0.003$ ) and previous experience $(p=0.008)$ were factors that influenced their fidelity. The qualitative data also postulated participant-level (eg, individual needs) and programme-level factors (eg, resources) as additional elements that influenced fidelity.

Conclusion The intervention was delivered with high fidelity. This study contributes to the limited evidence regarding fidelity assessment methods within complex behaviour change interventions. The findings suggest a combination of quantitative methods is suitable for the assessment of fidelity of delivery. A mixed methods
Strengths and limitations of this study

- This mixed methods investigation of fidelity of delivery and its influencing factors provides valuable information on fidelity assessment methods and factors to be considered in developing and evaluating complex behaviour change interventions.

- The novel use of mixed methods to assess fidelity in this study enabled increased certainty in findings where qualitative data corroborated the quantitative results.

- This study does not explore the fidelity of the quality of delivery (eg, therapist competence) or specific behaviour change techniques which will be reported in a separate publication.

approach provided a more insightful understanding of fidelity and its influencing factors.

Trial registration number ISRCTN49875385; Pre-results.

\section{INTRODUCTION}

Fidelity of delivery is an important, yet often overlooked aspect of behaviour change interventions and has been defined as the degree to which an intervention, treatment or programme is delivered as intended by the intervention developers. ${ }^{1}{ }^{2}$ Without adequately addressing fidelity in behaviour change research, it is uncertain that changes observed in study outcomes are due to the influence of the independent variable (the intervention being investigated) and not due to variability in its implementation, for example, extraneous elements that may have been added (either accidentally or purposely), or essential elements of the intervention that were omitted. ${ }^{3}$ In particular, as behaviour change interventions are often complex interventions that typically involve several components with the potential to affect or influence outcomes separately, it 
is especially important to incorporate adequate fidelity planning and assessment into the development of interventions of this nature. ${ }^{4}$

Despite a recent increased emphasis on improved assessment and reporting of what happens within complex behaviour change interventions, ${ }^{56}$ fidelity is still poorly addressed within this context, with few examples of fidelity being assessed comprehensively or reported adequately. ${ }^{7-9}$ Where studies have assessed fidelity within a behaviour change healthcare context, there is often limited exploration of the factors that might have influenced that fidelity. ${ }^{10}{ }^{11}$ Previous work that has specifically examined influencing factors in areas of public health, obesity and stroke research found that provider-level variables, such as experience, knowledge or skills, may influence fidelity of delivery. ${ }^{12-15}$ Although the use of both quantitative and qualitative methods has been previously recommended to comprehensively assess fidelity, ${ }^{16-18}$ this guidance is not consistently followed. Consequently, the use of quantitative methods in isolation may not allow for exploration of the factors influencing fidelity, knowledge of which could improve how fidelity is enhanced and assessed in future similar interventions. ${ }^{19}$ For example, French et $a l^{11}$ used audio-recordings and self-report methods to assess the fidelity of delivery of an educational intervention for general practitioners. The authors acknowledged that the quantitative study design did not allow them to explore the reasons for variations in fidelity scores found.

According to existing guidelines for addressing fidelity within behaviour change research developed by the National Institutes of Health Behaviour Change Consortium, ${ }^{18}{ }^{20}$ the fidelity of delivery of the intervention by providers is one particularly important aspect of fidelity. This aspect considers strategies that enhance the fidelity of delivery (eg, using treatment manuals or intervention protocols) and methods that assess this delivery (eg, provider self-report, audio or video-recorded observations and direct in vivo observations). However, although previous research has advocated a combination of these strategies in order to assess fidelity in-depth, ${ }^{21}{ }^{22}$ limited examples exist within the literature. Additionally, few studies have explored the relationship between these methods, and the accuracy of potentially more feasible methods against the 'gold standard' of direct observations using prespecified criteria ${ }^{23}$ has been poorly investigated. $^{181924}$ As a result, there is little evidence to justify the selection of one method over another or to inform the use of multiple methods simultaneously.

The current study is set within the context of the Self-Management of Osteoarthritis and Low back pain through Activities and Skills (SOLAS) feasibility trial (ISRCTN49875385).$^{25}$ The trial aims to evaluate the feasibility of providing the SOLAS intervention (experimental group) to promote self-management for patients with osteoarthritis of the hip/ knee and/or chronic low back pain compared with usual physiotherapy, which will serve as the pragmatic control group in order to determine the feasibility of moving to a full scale trial by following the Medical Research Council (MRC) guidelines. The intervention consists of sixweekly sessions of $90 \mathrm{~min}$ to be delivered by a primary care physiotherapist to a group of 6-8 people. Each session is divided into education and exercise sections (each approximately $45 \mathrm{~min}$ in duration). During the exercise section, participants are provided with an opportunity to attempt and practice prespecified exercises, while the education section is further split into four categories: Materials-participants are provided with materials intended to supplement and enhance understanding and uptake of skills; Introduction and review-at the start of each session, the physiotherapist introduces session aims and reviews goals and action plans with participants; Education-the physiotherapist facilitates a group discussion on targeted self-management skill or behaviour of the session using Powerpoint slides and projector; Review and planning-before the session concludes, the physiotherapist recaps participants' planned activity levels and action plans. For each category, a number of components (ie, specific elements or activities which varied according to the session) were intended to be delivered or addressed during the session by the physiotherapist (eg, provision of pedometers, provision of information on balanced weight and healthy eating), as detailed in an intervention manual ${ }^{2526}$ (summarised in table 1). Prior to participation, physiotherapists were provided with the manual during a 2-day training course where background variables were collected, in addition to a post-training assessment. ${ }^{26}$ Details of the development and testing of the fidelity protocol used in this study are described elsewhere. ${ }^{27}$ This study also does not explore the fidelity of the quality of delivery (eg, therapist competence) or specific behaviour change techniques (BCTs) which will be reported elsewhere.

The aim of this study was to establish the fidelity of delivery of a complex behaviour change intervention and the reasons for these findings using a mixed methods approach. Specifically, the study objectives were

1. to evaluate the agreement of multiple methods for assessing fidelity of delivery

2. to establish the fidelity of delivery of the SOLAS complex behaviour change intervention

3. to explore the potential factors that may have influenced these fidelity results.

\section{METHODS \\ Design}

This observational study was a convergent/triangulation mixed methods design. ${ }^{28}$ This mixed methods approach was chosen as it was felt that thorough integration of findings from both quantitative and qualitative methods would achieve a more comprehensive answer to the study questions by enabling the methods to be 'greater than just the sum of their parts'. ${ }^{29}$ Figure 1 graphically illustrates the study design, outlining the sequence of 


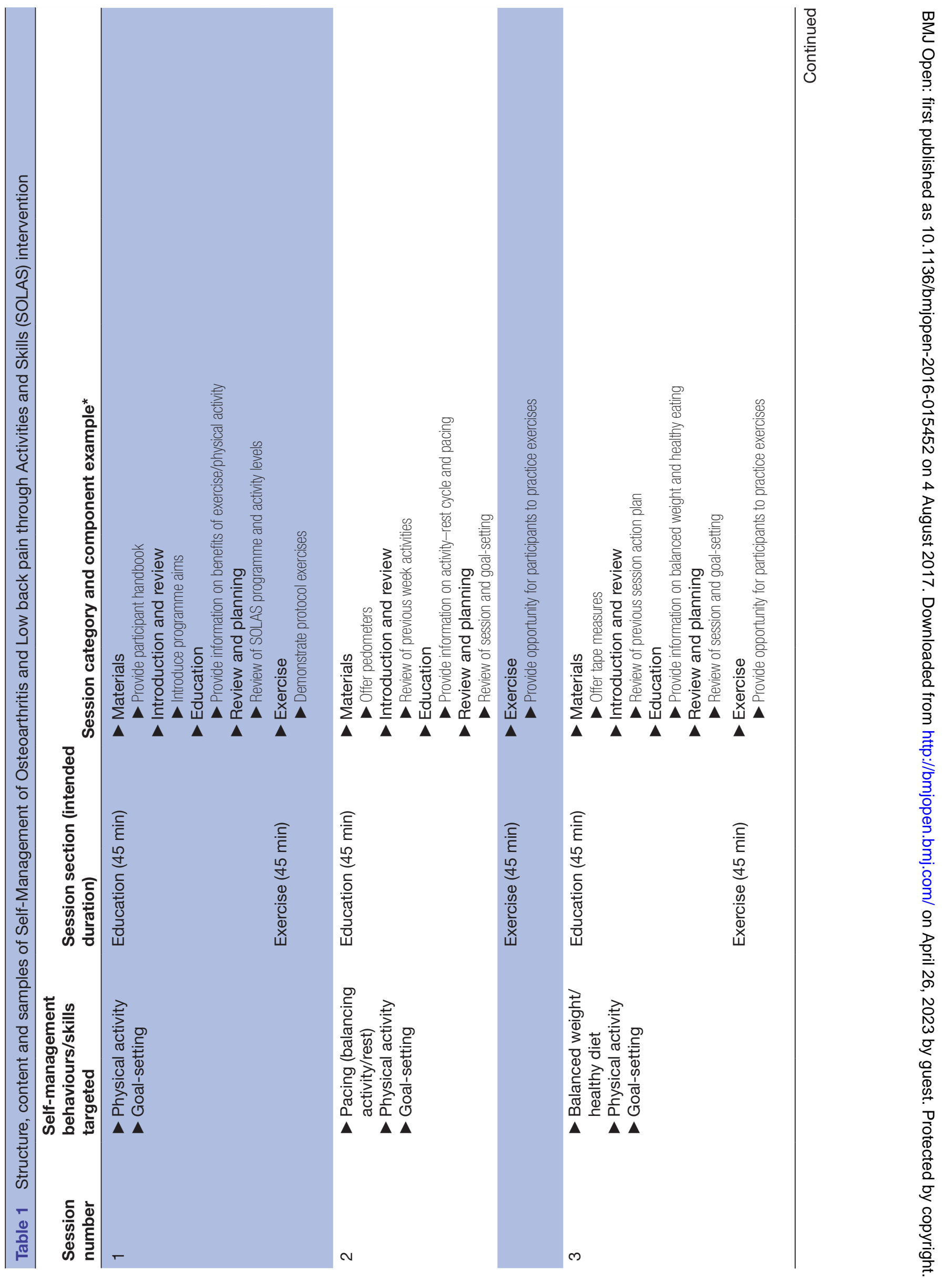



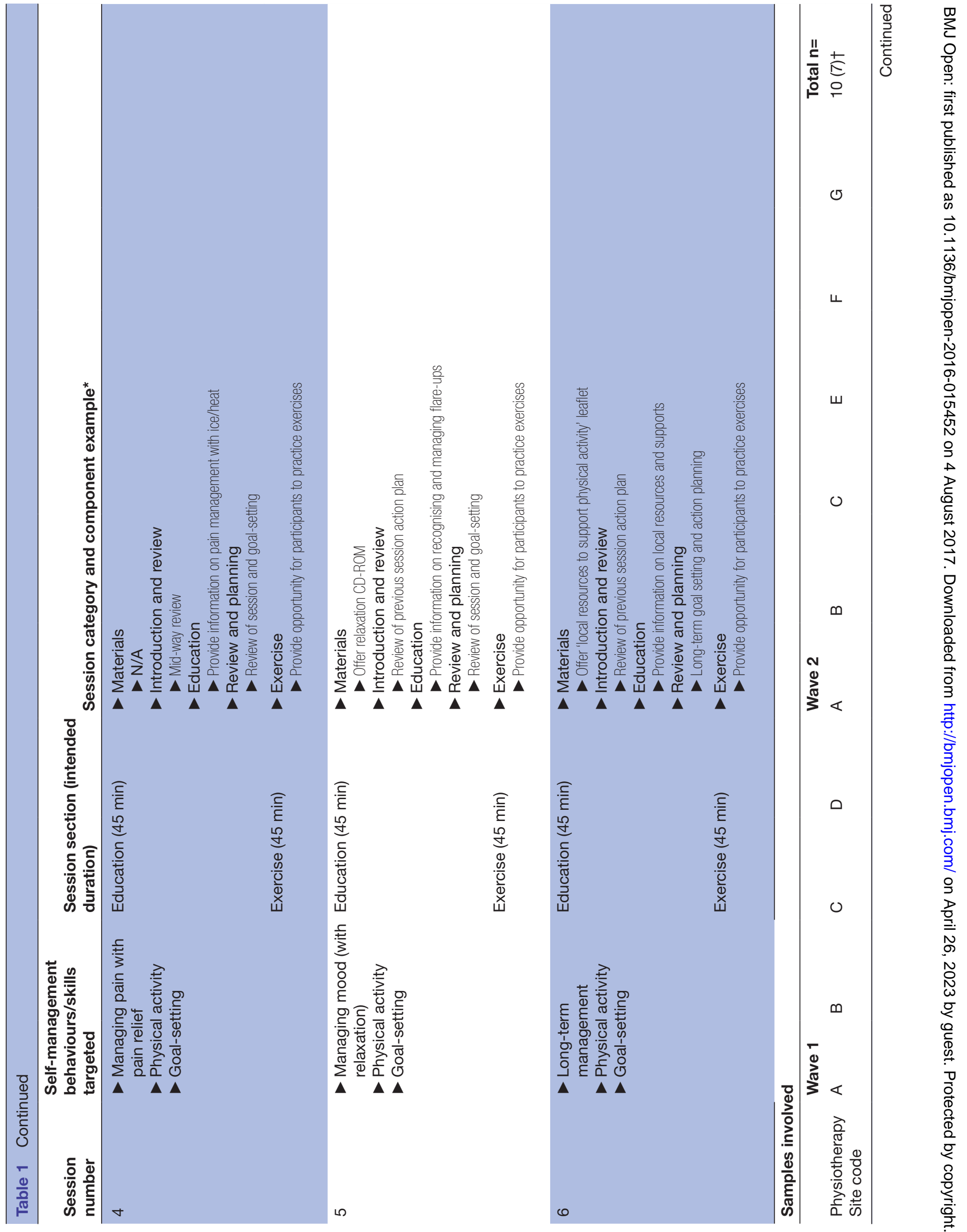


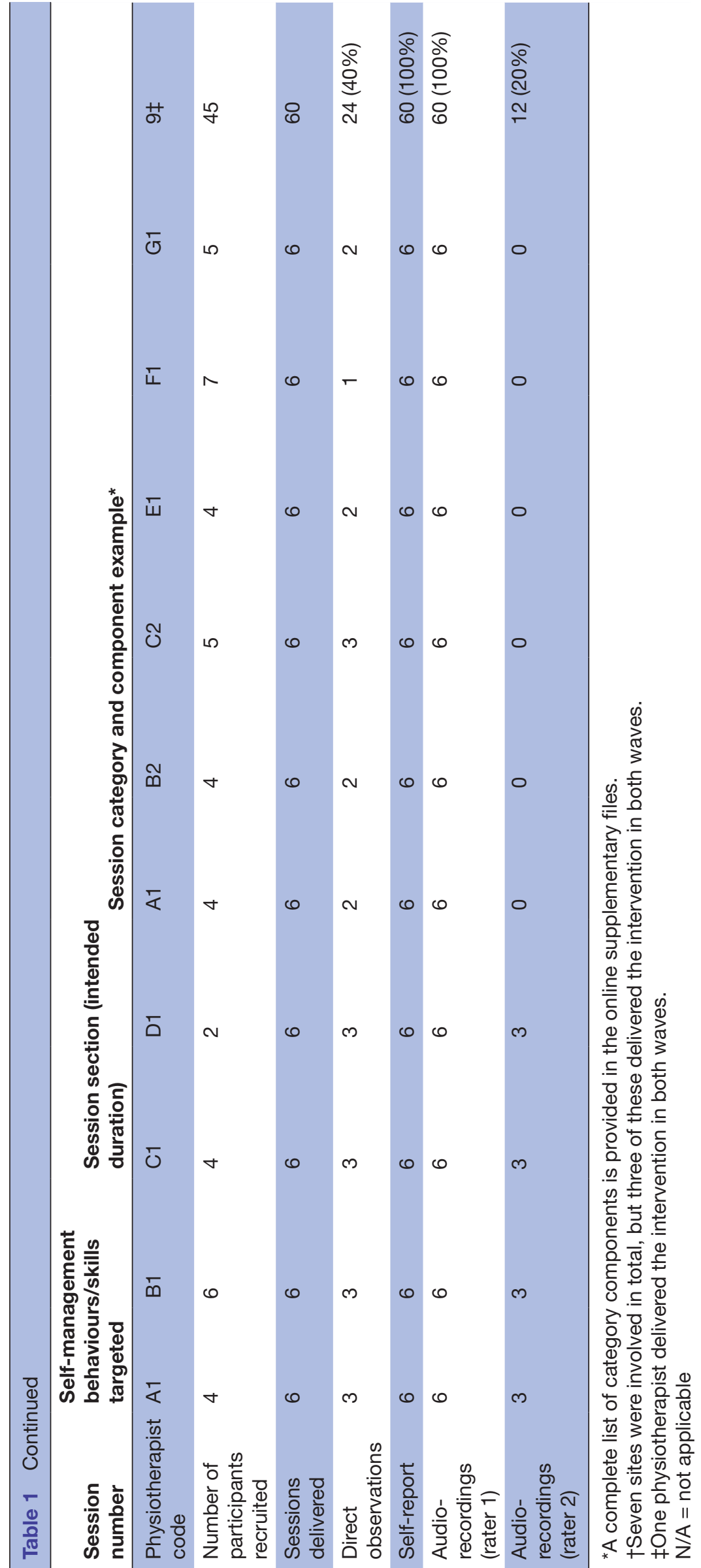




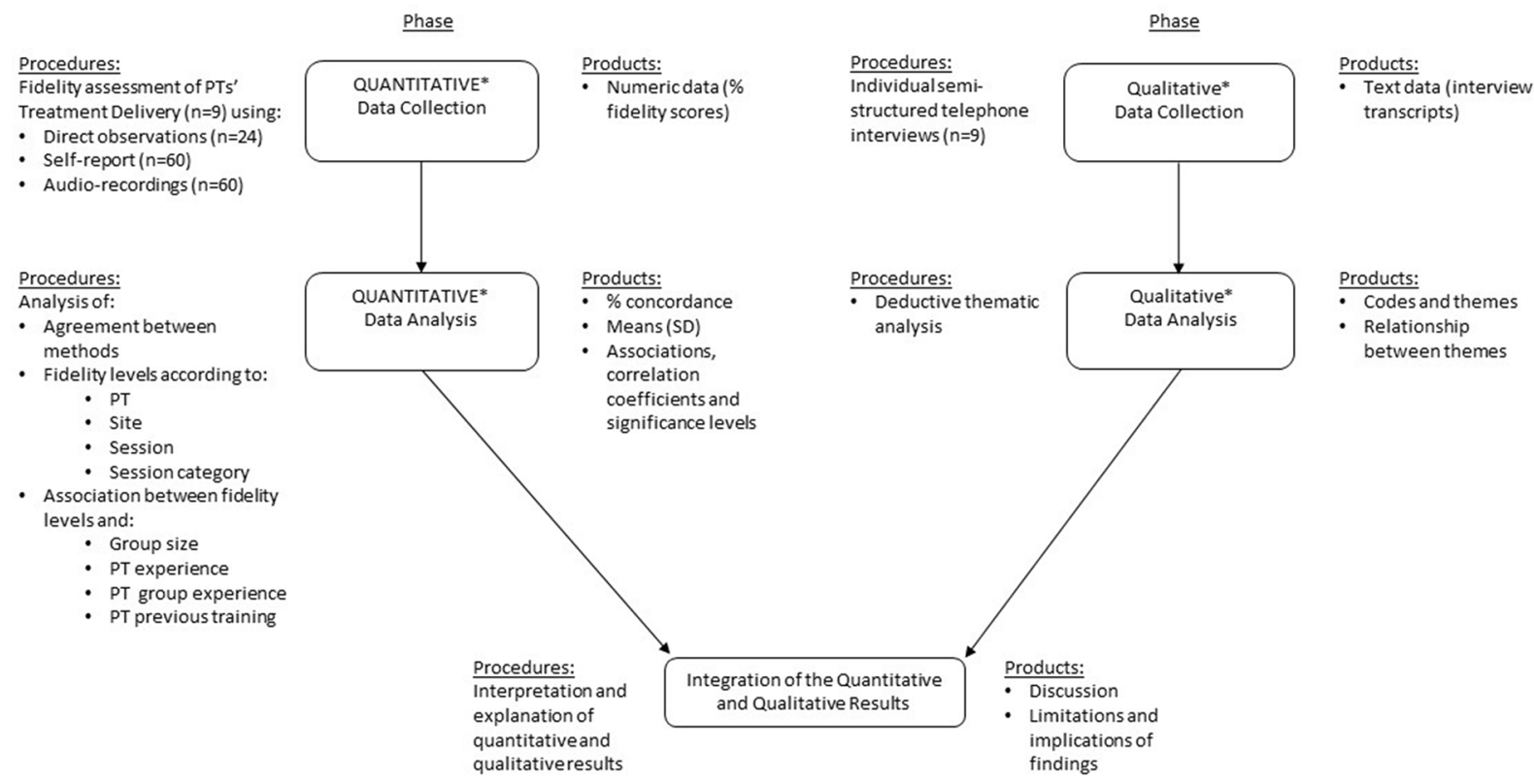

*capitalisation represents weighting or priority, $\mathrm{PT}=$ physiotherapist, $\mathrm{SD}=$ standard deviation

Figure 1 Diagrammatic representation of mixed methods convergent/triangulation design.

research activities, the priority of the methods and the stage at which integration occurred. ${ }^{30}$

\section{Quantitative phase}

Study sample and procedure

Data were collected during two of the three waves of the SOLAS feasibility trial (table 1), representing $71 \%$ of the overall trial data. Fidelity of delivery in this study refers to the assessment of both the delivery of session content, that is, providers deliver the session categories and components as intended (summarised in table 1) (fidelity of content) and session duration, that is, providers deliver the session as long as intended (fidelity of duration). Following pilot testing, it was decided to conduct 24 (40\% of sessions) randomly selected direct observations (rated by ET), 60 $(100 \%)$ self-report (rated by the physiotherapists) and 60 $(100 \%)$ audio-recordings (rated by ET) to assess fidelity of delivery using a priori checklists (see online supplementary file 1) that had been previously found to be feasible for use. ${ }^{27}$ To assess inter-rater reliability, 12 sessions $(20 \%)$ were rated by a second independent rater (AK). ${ }^{10}$ Checklists consisted of approximately 25 components for each session, structured according to the SOLAS categories. Components for each session were chosen to address each element specified in the SOLAS intervention manual (summarised in table 1$)^{25} 26$ to be delivered during that session. Each component was rated as 'yes/ present' equating to a score of two points, 'no/absent' (zero points) or 'attempted' (one point). Session duration was documented by all methods, and attendance was recorded by self-report.
Data analysis

Fidelity data analysis was consistent with standard procedures $^{19} 3132$ using SPSS V.20. Specifically, levels of agreement between methods and inter-rater reliability of audio-recorded data were assessed using percentage concordance. Overall mean fidelity of content scores (ie, percentage of manual-specified components delivered as intended) and fidelity scores according to physiotherapy site, physiotherapist, session and session category were obtained by calculating total actual scores as a percentage of the total possible score. Means data were compared using analysis of variances and Kruskal-Wallis tests. Fidelity of duration was established by calculating the difference between the actual and the intended session duration using a one-sample Wilcoxon test. Levels of fidelity were interpreted as previously reported in the literature, with $80 \%-100 \%$ adherence interpreted as 'high' fidelity, $51 \%-79 \%$ as 'moderate' and $0 \%-50 \%$ as 'low' fidelity. ${ }^{33334}$ Finally, the relationship between fidelity scores and (1) the number of participants present (group size) and (2) physiotherapist variables, that is, experience (years qualified), group experience (years delivering group physiotherapy), knowledge of intervention (posttraining evaluation score) and previous relevant training (see online supplementary file 2), were calculated using Spearman's correlation coefficient and Mann-Whitney $\mathrm{U}$ test. These physiotherapist variables were chosen for reasons described in the introduction.

\section{Qualitative phase}

The aim of the qualitative phase was to explore physiotherapists' opinions of fidelity of intervention delivery 
and the factors that they felt may have influenced their fidelity. Individual semistructured telephone interviews were conducted by an experienced qualitative researcher (SG) with each physiotherapist ( $\mathrm{n}=9$ ) within 2 weeks of intervention delivery completion. A topic guide with specific questions and probes related to fidelity was developed by the corresponding author (ET) (see online supplementary file 3). Interviews were audio-recorded and transcribed verbatim. Deductive thematic analysis was used to analyse the interviews as it is a flexible method that works with a range of research questions, including understanding people's experiences of programmes and healthcare interventions. ${ }^{35}$

Meaningful units of text were highlighted within each interview, then summarised and coded. Codes dealing with similar issues were grouped across all interviews and refined into themes. The reliability of themes was established by a second reviewer (AK), who independently analysed a randomly selected sample of $50 \%$ of the transcript extracts using the coding framework. Percentage agreement was determined between the reviewers' respective coding of extracts. If agreement was $<70 \%$, consensus on conflicting decisions was obtained through discussion. $^{36}$

\section{Integration}

Integration of quantitative and qualitative data occurred at an interpretation level using triangulation methodology. Specifically, a meta-matrix was created to facilitate comparison of the findings. ${ }^{37}$ This involved presenting the quantitative data in a tabular format alongside summarised qualitative themes, which enabled a transparent approach to determining convergence, discrepancy or silence across the findings of the data sets. ${ }^{38}$ Convergence was defined as general agreement between the data sets on the element of comparison (eg, overall quantitative fidelity score compared with the majority of physiotherapist opinions of their fidelity levels), while discrepancy was defined as general disagreement between the data sets on the element of comparison. ${ }^{38}$ Silence was defined as where one set of results addressed a theme or example, but the other set of results did not yield any relevant data. ${ }^{38}$

\section{RESULTS \\ Quantitative findings \\ Agreement}

Agreement between direct observations and self-report was $74.6 \%, 75.4 \%$ between self-report and audio-recordings (rater 1) and $86.6 \%$ between direct observations and audio-recordings (rater 1). Inter-rater reliability of audio-recordings (rater 1 vs rater 2) was $81.3 \%$. Further detail is provided in online supplementary file 4.

\section{Fidelity of content}

Fidelity was found to be high in all assessment methods, with a mean score of $81.7 \%$ (range of $61.1 \%-95.8 \%$ ) for the audio-recordings, $92.7 \%(85.2 \%-96 \%)$ for the self-report and $82.7 \%(72.1 \%-100 \%)$ for the direct observations. Table 2 details the fidelity results for each method with scores $<80 \%$ (cut-off for 'high' fidelity) in bold. Significant differences between physiotherapists' individual fidelity scores were found. Fidelity scores were also found to differ significantly according to the session category (eg, the category 'Materials' was delivered with significantly less fidelity than the 'Education' category).

\section{Fidelity of duration}

All methods found a statistically significant difference between the actual duration of the exercise component and its intended duration of $45 \mathrm{~min}$ (see online supplementary file 5). When this was analysed for each individual session for all methods combined, a statistically significant difference $(p<0.001)$ was only found for session 1 between the actual and intended duration. The difference between the actual and intended duration for all other sessions was not found to be statistically significant ( $\mathrm{p}>0.05)$.

\section{Group size: participants in attendance}

The average number of participants present for all groups across all sessions was 3, with an SD of 1.3 (see online supplementary file 6). Overall, group attendance ranged from one to six participants for any session across both waves. The total number of participants recruited for each group was significantly different between physiotherapists (eg, seven participants were recruited for the group delivered by physiotherapist F1 compared with only two recruited for the group delivered by D1), as were the numbers of participants present (average group size). The size of groups did not differ significantly between sessions.

\section{Factors associated with fidelity}

Both direct observation and audio-recorded data showed a significant correlation between fidelity scores and the physiotherapists' post-training evaluation scores. Direct observation data also found a significant negative correlation between group sizes and the fidelity scores. Physiotherapist years qualified and experience of delivering groups were found to have significant, negative correlations with fidelity scores for the audio-recorded and self-report data respectively (table 3 ).

\section{Qualitative findings}

Inter-rater reliability of coding achieved $81.6 \%$ agreement. Overall, physiotherapists felt that they had delivered the programme with good fidelity. All physiotherapists discussed some deviations from the protocol or adaptations made during delivery, for example, goal-setting was found to be challenging to complete as intended. Other adaptations either concerned difficulties with use of programme materials (eg, using the projector) as intended or providing additional information during the education content. Five physiotherapists also discussed deviation from protocol in relation to duration, mostly during the first session, with one stating that her time management around the education wasn't always exactly what 
Table 2 Fidelity results calculated from \% totals

\begin{tabular}{|c|c|c|c|}
\hline & $\begin{array}{l}\text { Direct observations (DO) } \\
\% \text { (SD) }\end{array}$ & $\begin{array}{l}\text { Self-report (SR) } \\
\% \text { (SD) }\end{array}$ & $\begin{array}{l}\text { Audio } 1 \text { (AO) } \\
\% \text { (SD) }\end{array}$ \\
\hline Total \% mean fidelity score (SD) & $82.7 \%(10)$ & $92.7 \%(6.4)$ & $81.7 \%(12)$ \\
\hline \multicolumn{4}{|l|}{$\%$ mean fidelity score per session $(\mathrm{SD})^{\star}$} \\
\hline 1 & $88.8 \%(5.24)$ & $95 \%(4.5)$ & $91.6 \%(4.5)$ \\
\hline 2 & $82.8 \%(5.7)$ & $92 \%(6.9)$ & $86.8 \%(10.5)$ \\
\hline 3 & $85.6 \%(12.9)$ & $96 \%(4.2)$ & $81.4 \%(10.6)$ \\
\hline 4 & $83.3 \%(14.4)$ & $90.9 \%(8.3)$ & $75 \%(14)$ \\
\hline 5 & $74.1 \%(11.9)$ & $89.4 \%(8.1)$ & $78.7 \%(11)$ \\
\hline 6 & $82 \%(9.6)$ & $92.7 \%(3.78)$ & $74.9 \%(12)$ \\
\hline \multicolumn{4}{|l|}{$\%$ mean fidelity score per site $(\mathrm{SD})^{*}$} \\
\hline A (delivered twice, same physiotherapist) & $78.7 \%(7.6)$ & $95 \%(5.4)$ & $81.3 \%(11.5)$ \\
\hline B (delivered twice, two physiotherapists) & $76.7 \%(5.6)$ & $92.8 \%(5.3)$ & $71.1 \%(10)$ \\
\hline C (delivered twice, two physiotherapists) & $84.8 \%(11.8)$ & $91.8 \%(7.7)$ & $84.9 \%(8.1)$ \\
\hline $\mathrm{D}$ & $87.2 \%(4)$ & $93.2 \%(2.9)$ & $87.1 \%(4.4)$ \\
\hline $\mathrm{E}$ & $83.1 \%(13)$ & $94.3 \%(3.8)$ & $88.3 \%(8.7)$ \\
\hline $\mathrm{F}$ & $72.5 \%$ & $85.2 \%(9.6)$ & $72.9 \%(15)$ \\
\hline G & $100 \%(0)$ & $94.7 \%(4.4)$ & $92.8 \%(5.4)$ \\
\hline \multicolumn{4}{|l|}{$\%$ mean fidelity score per category (SD) $\dagger$} \\
\hline Materials & $72.1 \%(19.4)$ & $86 \%(17)$ & $61.1 \%(29.6)$ \\
\hline Introduction and review & $82.6 \%(16.3)$ & $92.9 \%(12.8)$ & $76.2 \%(24.5)$ \\
\hline Education & $93.3 \%(8.6)$ & $97.1 \%(6.6)$ & $95.4 \%(6.9)$ \\
\hline Exercise & $80.4 \%(14)$ & $95.4 \%(7.1)$ & $82.4 \%(13)$ \\
\hline Review and planning & $77.1 \%(33)$ & $90.8 \%(21.6)$ & $69.8 \%(39.6)$ \\
\hline \multicolumn{4}{|l|}{ \% mean fidelity score per physio (SD)‡ } \\
\hline A1 & $78.8 \%(7.6)$ & $95.1 \%(5.4)$ & $81.3 \%(11.5)$ \\
\hline B1 & $76.1 \%(7.9)$ & $92.2 \%(7.2)$ & $72.3 \%(9.3)$ \\
\hline B2 & $77.5 \%(0.4)$ & $93.4 \%(3)$ & $72.6 \%(12.5)$ \\
\hline $\mathrm{C} 1$ & $93.4 \%(2)$ & $85.2 \%(4.6)$ & $91 \%(3.5)$ \\
\hline $\mathrm{C} 2$ & $76.2 \%(11)$ & $98.5 \%(1.9)$ & $78.8 \%(6.6)$ \\
\hline D1 & $87.2 \%(4)$ & $93.2 \%(2.9)$ & $87.1 \%(4.4)$ \\
\hline E1 & $83.1 \%(13)$ & $94.3 \%(3.8)$ & $88.3 \%(8.7)$ \\
\hline $\mathrm{F} 1$ & $72.5 \%$ & $85.2 \%(9.6)$ & $69.8 \%(14.7)$ \\
\hline G1 & $100 \%(0)$ & $94.7 \%(4.4)$ & $95.8 \%(5.4)$ \\
\hline
\end{tabular}

Bold values $=$ scores $<80 \%$.

*Significant differences between sites $(p<0.001)$ and between sessions $(p=0.007)$ according to $A O$, not significantly different according to $D O$ and SR.

†Significant differences between categories according to DO ( $p=0.007)$, SR $(p<0.001)$ and $A O(p<0.001)$.

fSignificant differences between physiotherapists according to DO $(p=0.019)$, SR $(p=0.004)$ and AO $(p<0.001)$.

it should have been' (B1, transcript line 278-286). In terms of factors that influenced fidelity (ie, reasons discussed for the aforementioned adaptations and deviations), six themes were found that were structured into three levels of factors-physiotherapist, participant and programme level (figure 2).

At the physiotherapist level, eight of the nine physiotherapists felt that knowledge of the content of the SOLAS programme facilitated their delivery of the education session and made it easier. Conversely, deviations from protocol within the education content discussed by six physiotherapists were due to the provision of additional information which was influenced by their previous experiences of delivering similar groups (eg, talking more about pain pathophysiology because of previous classes delivered on this topic). This formed the theme 'Physiotherapist knowledge and experience influenced delivery of SOLAS-education content'.

At the participant level, five physiotherapists felt that participants' individual needs such as their understanding 
Table 3 Association between fidelity scores and variables

\begin{tabular}{|c|c|c|c|c|}
\hline Variable & Direct observations & Self-report & Audio-recordings & Statistical test \\
\hline Group size (number present) & $-0.434(p=0.034)^{\star}$ & $-0.215(p=0.98)$ & $-0.193(p=0.151)$ & \multirow{4}{*}{$\begin{array}{l}\text { Spearman's r ( } p \\
\text { value) }\end{array}$} \\
\hline $\begin{array}{l}\text { Physiotherapist experience } \\
\text { (years qualified) }\end{array}$ & $-0.09(p=0.676)$ & $-0.186(p=0.154)$ & $-0.346(p=0.008)^{\star}$ & \\
\hline $\begin{array}{l}\text { Physiotherapist group experience (years } \\
\text { delivering group physiotherapy) }\end{array}$ & $0.171(p=0.424)$ & $-0.364(p=0.004)^{\star \star}$ & $0.136(p=0.312)$ & \\
\hline $\begin{array}{l}\text { Physiotherapist post-training evaluation } \\
\text { score (\%) }\end{array}$ & $0.581(p=0.003)^{\star \star}$ & $-0.152(p=0.245)$ & $0.314(p=0.018)^{*}$ & \\
\hline $\begin{array}{l}\text { Physiotherapist previous relevant } \\
\text { training (yes/no) }\end{array}$ & $\mathrm{U}=33(\mathrm{p}=0.302)$ & $U=201(p=0.107)$ & $U=243(p=0.840)$ & $\begin{array}{l}\text { Mann-Whitney } U \\
\text { (p value) }\end{array}$ \\
\hline
\end{tabular}

${ }^{*} p$ is significant at $p<0.05,{ }^{* *} p<0.005$.

of content or language literacy levels influenced the delivery of education and exercise components and that adaptation sometimes occurred in response to these needs, creating the theme of 'Individual needs influenced delivery of SOLAS-education, exercise, goal-setting'. The number of participants present was discussed by seven physiotherapists as another participant-level factor that influenced fidelity of delivery and formed the participant-level theme of 'Group size influenced delivery of SOLAS— goal-setting, use of materials'. A further participant-level theme was 'Group dynamics influenced delivery of SOLAS_-goal-setting' as four physiotherapists felt that groups with good dynamics and interaction between participants led to better group discussions and better facilitation of goal setting.

The 'Amount of education content influenced delivery of SOLAS_duration'was a programme-level factor discussed by six physiotherapists, who felt that the amount of education content that was involved in the first session led to more time spent on the education aspect than intended as per protocol. Finally, all nine physiotherapists believed that the good resources (eg, booklets and handouts, venue space) enhanced and facilitated the delivery of the programme as intended and that occasionally poor or problematic resources (eg, lack of venue security) negatively influenced the delivery of the programme as intended. This created the theme 'Resources/materials influenced delivery of SOLAS_education and exercise content'. Exemplary quotes are provided in table 4.

\section{Integrating qualitative and quantitative findings}

Each theme was further analysed according to the quantitative data comparing physiotherapists who scored 'high' (ie, $\geq 80 \%$; physiotherapists D1, C1, E1 and G1) to those who scored 'moderate' (ie, $\geq 50 \%-79 \%$; B1, B2, C2 and F1). Physiotherapist A1 was not included in this analysis as her score was categorised as 'moderate' by direct observations and 'high' by audio-recorded data at $78.7 \%$ and $81.3 \%$, respectively. A difference between these
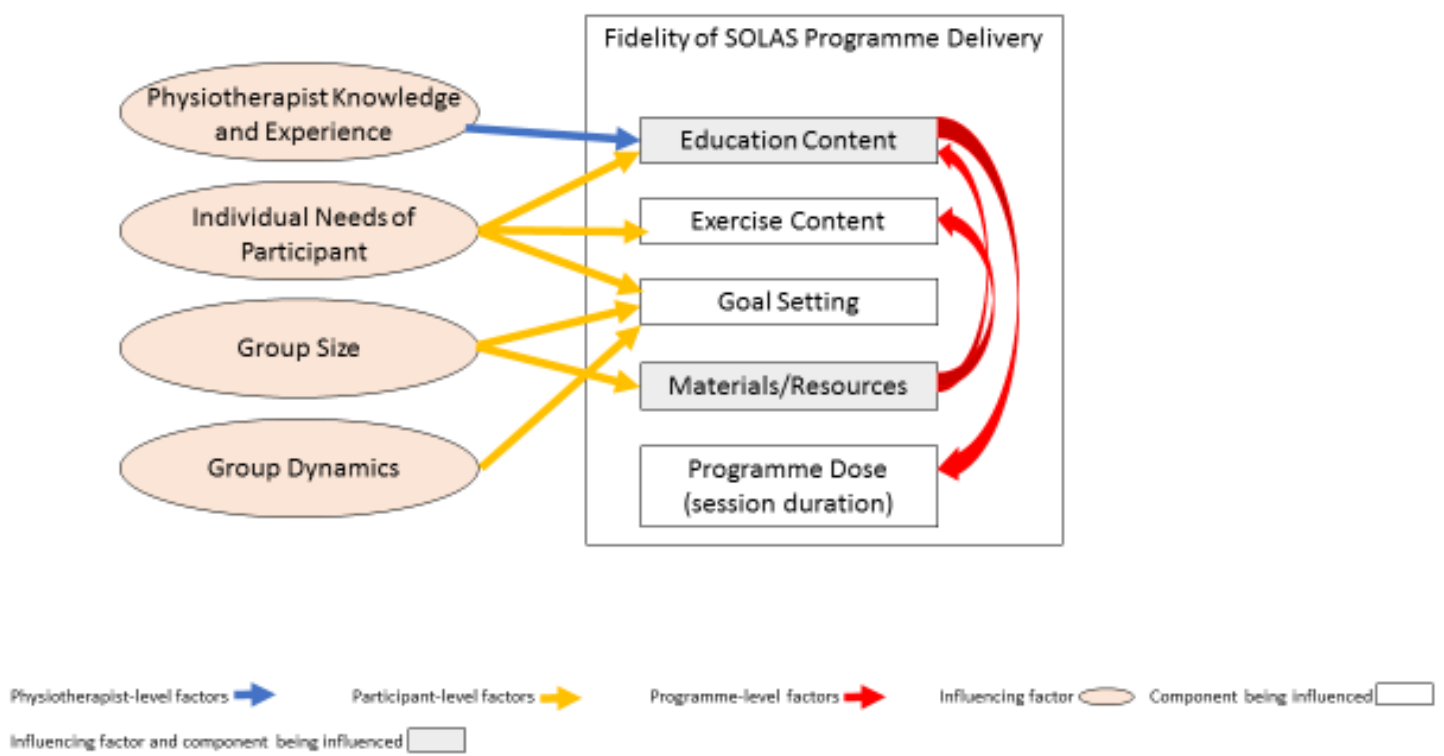

Figure 2 Visual representation of themes of qualitative interviews-factors influencing fidelity of Self-management of Osteoarthritis and Low back pain through Activity and Skills (SOLAS) delivery. 
Table 4 Qualitative findings of factors influencing fidelity results

\begin{tabular}{|c|c|c|}
\hline Factor level & Theme & Exemplary quote (physiotherapist code, transcript line) \\
\hline Physiotherapist & $\begin{array}{l}\text { Physiotherapist knowledge } \\
\text { and experience influenced } \\
\text { delivery of SOLAS- } \\
\text { education content }\end{array}$ & $\begin{array}{l}\text { 'In my previous experience I would have done a lot more actually on the pain } \\
\text { side of things... so in my previous class I would have had, you know, maybe } \\
\text { one full class on maybe pain perception and, kind of, the influence of emotion } \\
\text { and feelings ... so I think I would have probably maybe talked a lot more around } \\
\text { that pain section than maybe somebody else would have'. (C1, 75-99) }\end{array}$ \\
\hline Participant & $\begin{array}{l}\text { Individual needs influenced } \\
\text { delivery of SOLAS- } \\
\text { education, exercise, goal- } \\
\text { setting }\end{array}$ & $\begin{array}{l}\text { 'People don't like writing them [action plans] there and then you know with } \\
\text { pencils given and whatever-yes it's very hard to get people to write down } \\
\text { things like that ... . Where I work there is a lot of people health literacy is very } \\
\text { low ... so therefore that's a challenge for them...so I tend to be very careful } \\
\text { about pushing it out really'. (F1, 141-187) }\end{array}$ \\
\hline Participant & $\begin{array}{l}\text { Group size influenced } \\
\text { delivery of SOLAS - goal- } \\
\text { setting, use of materials }\end{array}$ & $\begin{array}{l}\text { 'The only thing I might find a little bit hard would be the goal setting. I suppose } \\
\text { you'd-that would be a bit more challenging because you'd have more } \\
\text { numbers in the group'. (G1,118-132) }\end{array}$ \\
\hline Programme & $\begin{array}{l}\text { Amount of education } \\
\text { content influenced delivery } \\
\text { of SOLAS-duration }\end{array}$ & $\begin{array}{l}\text { 'I found the content in week one was nearly too much ... by the time I finished } \\
\text { talking and ran through the exercises, the hour and a half was finished. And so } \\
\text { nobody actually practiced any of the exercises on the first day'. (B2, 96-106) }\end{array}$ \\
\hline Programme & $\begin{array}{l}\text { Resources/materials } \\
\text { influenced delivery of } \\
\text { SOLAS-education and } \\
\text { exercise content }\end{array}$ & $\begin{array}{l}\text { 'The slides didn't work for me this time ... .You can't lock that room .... once or } \\
\text { twice I didn't bring the laptop at all and I just had to print it out, all of the slides } \\
\text { on A4 laminate and so we talked all the slides ....' (F1, 207-240) } \\
\text { 'I think I only left out maybe three [exercise] stations, something like that. } \\
\text { Because we didn't have a bouncer and ... we didn't have a bed'. (C1, 113-121) } \\
\text { 'Nothing but positive feedback for all the content and the-the resources ... I just } \\
\text { think they complimented the-the education fantastically, I just thought they } \\
\text { added much more to the programme than not having these resources.' (E1, } \\
414-415)\end{array}$ \\
\hline
\end{tabular}

physiotherapist groups was found in only one theme, 'Group size influenced delivery of SOLAS'. Physiotherapists who scored higher on the fidelity assessments (average group size of 2.5 participants) believed it was easier to deliver goal-setting as intended with smaller groups. Conversely, physiotherapists with moderate fidelity scores (average group size of 3.7 participants) felt it was harder to facilitate goal-setting as intended with less numbers and believed it would be easier with bigger groups due to better engagement and group discussion. Further details of the triangulation are provided in table 5 where a meta-matrix was used to compare between findings from both data sets. For the most part, convergence was found between the qualitative and quantitative data, though four qualitative themes relating to influencing factors had no corresponding quantitative data (silence). No areas of discrepancy were found.

\section{DISCUSSION}

The aim of this mixed methods study was to explore and evaluate fidelity of delivery within a feasibility trial of a complex behaviour change intervention using multiple assessment methods. The study found good agreement between researcher-delivered direct observation and audio-recorded fidelity assessment methods, with lower agreement found between provider self-report and researcher-delivered methods. The intervention content was delivered overall with high fidelity, with some variation between physiotherapists and between certain intervention categories. The intervention duration was found to have deviated significantly from intended during the first session only. Subsequently, qualitative interviews with physiotherapists confirmed these fidelity findings. Finally, both qualitative and quantitative data showed that physiotherapists' knowledge and previous experience, as well as the group size, were factors that influenced their fidelity of intervention delivery. The qualitative data contributed further, and postulated additional participant and programme-level factors as aspects that also influenced the overall fidelity results.

Agreement between direct observations and audio-recordings for assessing the fidelity of delivery was found to be excellent. ${ }^{39}$ Agreement between both of these methods and provider self-report assessment was lower, as providers consistently rated themselves higher than the independent raters. These findings are perhaps unsurprising as both direct observations and audio-recordings were rated by the same researcher, and numerous previous studies have shown that providers' subjective assessments of fidelity are often rated higher than independent assessments. ${ }^{32}{ }^{32}$ Taking direct 


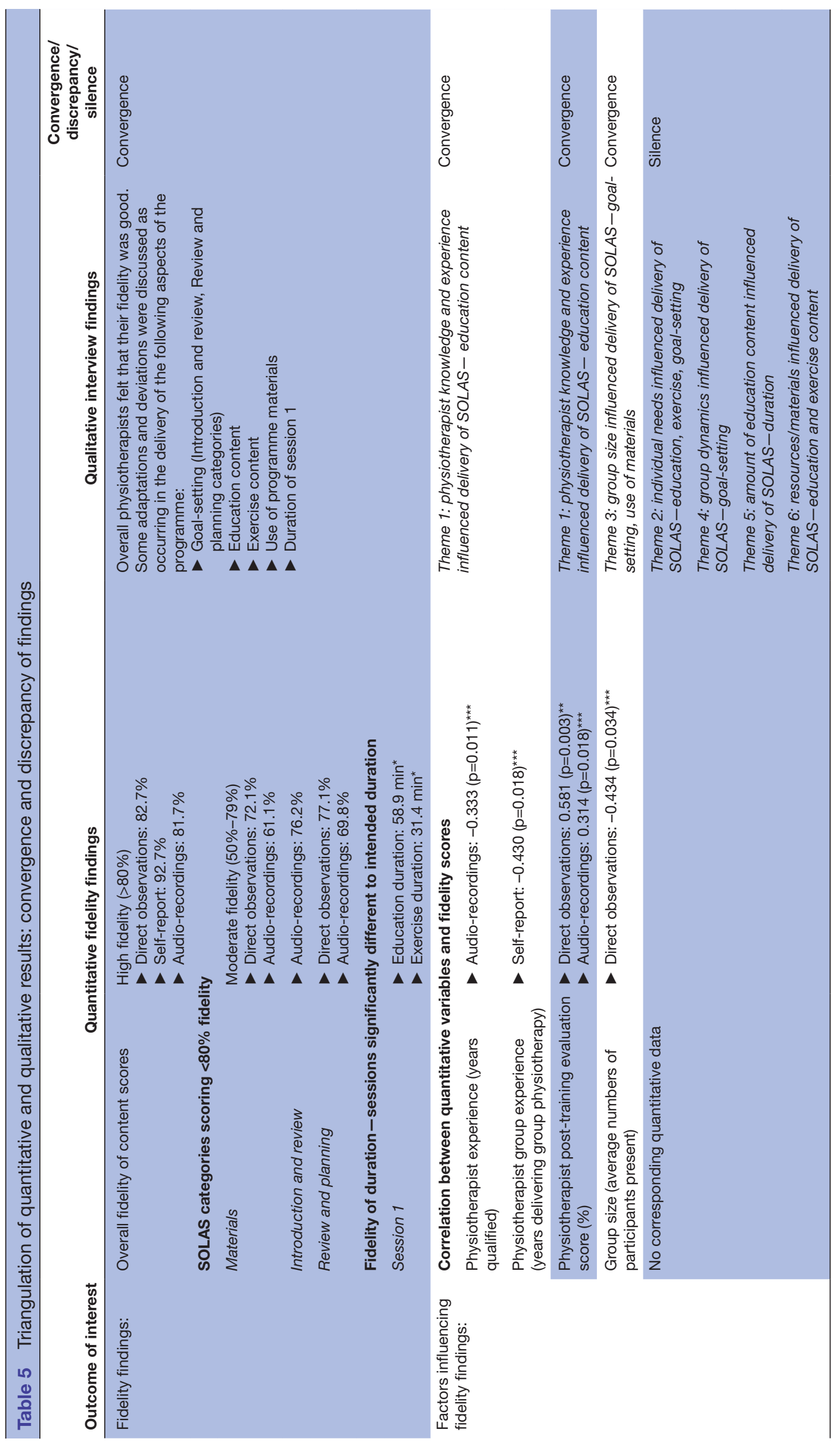

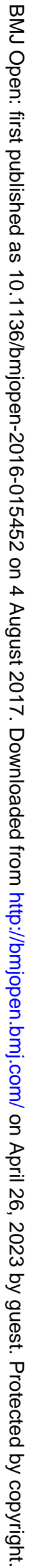


observations as the commonly cited 'gold standard', 11820 these findings reinforce that self-report methods may not be the most accurate method for assessing fidelity in a complex behaviour change study. However, they may still have their place for recording data and also for enhancing fidelity to the protocol by serving as an aide memoire for providers. ${ }^{41}$ Although direct observations and audio-recordings have their own limitations, ${ }^{24}{ }^{42}$ previous piloting of these assessment methods found that they were feasible and acceptable to physiotherapists. ${ }^{27}$ Additionally, the good agreement between audio-recordings and direct observations found in this study suggests that audio-recordings may be a viable alternative with limited resources, as has been done in similar interventions. ${ }^{43}$ However, where resources allow, a combination of multiple quantitative methods may provide the most in-depth assessment of fidelity.

One of the key study findings of this study was reinforcing the value of using mixed methods research for the assessment of fidelity. This approach was emphasised in the recent MRC guidelines for conducting process evaluations of complex interventions ${ }^{6}$ and is becoming increasingly used in the widespread implementation of evidence-based interventions ${ }^{44}$ but does not yet appear to be common practice within fidelity assessments of behaviour change interventions. ${ }^{10} 21$ The integration of quantitative and qualitative results enabled the triangulation of findings to provide a better overall picture of the fidelity of the SOLAS intervention and its influencing factors. The importance of the qualitative contribution to answering the "why' question is evident in the fact that the physiotherapist interviews unearthed strong participant and programme-level factors associated with fidelity results that were not apparent from the quantitative data alone. While this may be predominantly due to the focus of the quantitative analysis on physiotherapist-level variables which were chosen based on existing literature, the participant and programme-level factors identified by this analysis such as group dynamics or amount of programme content may have been difficult to quantitatively analyse to demonstrate association with fidelity results.

This study found that the factors that may influence the fidelity of an interventions' delivery can occur on three levels : provider, participant and programme. Where previous studies have explored factors that have influenced fidelity of intervention delivery, many have focused solely on provider-level factors, demonstrating associations between fidelity and factors such as provider training or skills. ${ }^{13} 192145$ The findings of this study have valuable implications for future studies that aim to assess and enhance fidelity of similar interventions as they indicate that planning for fidelity should include considering potential influencing factors at each of these three levels. These results are consistent with recent conclusions by Masterson-Algar et al in a stroke rehabilitation setting, ${ }^{14}$ who found that investigating fidelity within clinical trials should also take the individual needs of patients into account, and also concur with the findings of an education-based intervention that found the most common reason for adaptation within intervention delivery was insufficient time. $^{46}$

On the physiotherapist-level, better knowledge of the intervention content and structure was found to positively correlate with quantitative fidelity scores, with a causative link established via the qualitative investigation. This echoes previous findings by Huijg et al, who showed that physiotherapist skill level was one of the most important predictors of fidelity. ${ }^{21}$ A more targeted approach to enhancing fidelity in future interventions may therefore be warranted, such as identifying physiotherapists at higher risk of lower fidelity using post-training evaluation scores and employing more focused fidelity assessment of delivery or further training for these providers, ${ }^{13}$ as has been previously employed in similar interventions. ${ }^{1043}$ The results of the study also showed that physiotherapists with more experience of certain aspects tended to emphasise these at the expense of delivering other components as comprehensively as intended in the protocol. These experience-based adaptations invoke the well-established issue of adaptation versus fidelity. For years, research has debated the concept of fidelity versus adaptation, with the case made for both strict fidelity and for modifying interventions. ${ }^{47} \mathrm{~A}$ third view is that both fidelity and adaptation are essential, and achieving an appropriate balance between both can allow an intervention to maximise its effectiveness, while being generalisable and flexible enough to be implementable. ${ }^{49}$ To achieve this, our fidelity checklists included components that encouraged elements of treatment individualisation (eg, individualised feedback regarding exercises). However, it may be that these checklists still did not allow for enough individualisation within delivery, an aspect that should be considered by other researchers seeking to conduct similar fidelity assessments.

A limitation of this study was the timing of the interviews, which did not allow a 'pure' convergent/ triangulation design. Typically, the qualitative and quantitative methods occur concurrently in this design ${ }^{28}$ however, they were scheduled to take place after physiotherapists had experienced delivery of an entire 6-week SOLAS intervention. Although a sequential explanatory design ${ }^{30}$ where quantitative results were analysed prior to completing the interviews might have enabled further probing of the factors influencing fidelity, interviews were conducted within 2 weeks of the intervention completion to minimise recall bias. Due to time constraints, it was not possible to have the quantitative data collected and analysed beforehand. Finally, this study mostly focuses on the adherence of delivery (eg, intervention content and duration) and does not address the quality or competence of delivery of SOLAS (eg, interpersonal or communication style of the physiotherapist), or use of specific BCTs, which is being addressed in a separate publication. This study also does not examine the broader aspects of fidelity 
such as provider training or participant receipt as these were beyond the scope of this publication and will be addressed in a future paper.

\section{CONCLUSIONS}

In process evaluations and fidelity assessments of largescale complex interventions, it is often recommended to complete and report the results of the fidelity assessment before the trial outcomes so as not to bias reporting. ${ }^{50}$ Future work will investigate the relationship between this evaluation of fidelity of delivery, the SOLAS feasibility trial outcomes (analysis currently under way) and the evaluation of fidelity of BCT delivery, enabling a potentially more insightful and accurate interpretation of findings. This study also has valuable implications for further research and the overall science of fidelity as it contributes much needed information to the limited current evidence for the application of fidelity assessment methods within the area of complex behaviour change. The findings have demonstrated how multiple quantitative methods can be used to assess the fidelity of delivery of a complex behaviour change intervention, and that a combination of methods may be most suitable, depending on their acceptability and available resources. We have also shown how the use of a mixed methods approach, integrating both quantitative and qualitative data, provides a more insightful understanding of the factors influencing fidelity.

Acknowledgements The authors thank the physiotherapists and participants for taking part in the study. They also like to thank Alison Keogh for her help in the inter-rater reliability analysis of the audio-recorded data and physiotherapist interviews and Associate Professor Suzanne Guerin for conducting the physiotherapist interviews.

Contributors ET conceptualised and designed the study, conductedthe quantitative data collection and completed the study analysis andinterpretation. JM and DAH provided guidance on the design. DAH is the principal investigator of the SOLAS trial and obtained funding for this study within the trial. All authors provided methodological consultation and were involved in substantial contributions to reviewing of the drafts. All authors approved the final version of the manuscript.

Funding This study was funded as part of Health Research Award HRA HSR/2012/24 by the Health Research Board of Ireland. The funding source had no other involvement in this publication.

Competing interests None declared.

Patient consent Obtained.

Ethics approval University College Dublin Human Research Ethics Committee (LS-13-54).

Provenance and peer review Not commissioned; externally peer reviewed.

Data sharing statement Quantitative checklists, the qualitative topic guide and detailed data tables have been provided in the supplementary files. Further information is available if required from the corresponding author at elaine. toomey@nuigalway.ie.

Open Access This is an Open Access article distributed in accordance with the Creative Commons Attribution Non Commercial (CC BY-NC 4.0) license, which permits others to distribute, remix, adapt, build upon this work non-commercially, and license their derivative works on different terms, provided the original work is properly cited and the use is non-commercial. See: http://creativecommons.org/ licenses/by-nc/4.0/

(c) Article author(s) (or their employer(s) unless otherwise stated in the text of the article) 2017. All rights reserved. No commercial use is permitted unless otherwise expressly granted.

\section{REFERENCES}

1. Gearing RE, El-Bassel N, Ghesquiere A, et al. Major ingredients of fidelity: a review and scientific guide to improving quality of intervention research implementation. Clin Psychol Rev 2011;31:79-88.

2. Carroll C, Patterson M, Wood S, et al. A conceptual framework for implementation fidelity. Implementation Science 2007;2:1-9.

3. Borrelli B, Sepinwall D, Ernst D, et al. A new tool to assess treatment fidelity and evaluation of treatment fidelity across 10 years of health behavior research. J Consult Clin Psychol 2005;73:852-60.

4. Craig P, Dieppe P, Macintyre S, et al. Developing and evaluating complex interventions: the New Medical Research Council guidance. BMJ 2008;3370:a1655-6.

5. Hoffmann TC, Glasziou PP, Boutron I, et al. Better reporting of interventions: template for intervention description and replication (TIDieR) checklist and guide. BMJ 2014;348:g1687.

6. Moore GF, Audrey S, Barker M, et al; Process evaluation of complex interventions: medical Research Council guidance, 2015.

7. Hoffmann TC, Walker MF, Langhorne P, et al. What's in a name? the challenge of describing interventions in systematic reviews: analysis of a random sample of reviews of non-pharmacological stroke interventions. BMJ Open 2015;5:e009051.

8. Schinckus L, Van den Broucke S, Housiaux M; Diabetes Literacy Consortium. Assessment of implementation fidelity in diabetes selfmanagement education programs: a systematic review. Patient Educ Couns 2014;96:13-21.

9. Toomey E, Currie-Murphy L, Matthews J, et al. Implementation fidelity of physiotherapist-delivered group education and exercise interventions to promote self-management in people with osteoarthritis and chronic low back pain: a rapid review part II. Man Ther 2015;20:287-94.

10. Mars T, Ellard D, Carnes D, et al. Fidelity in complex behaviour change interventions: a standardised approach to evaluate intervention integrity. BMJ Open 2013;3:e003555.

11. French SD, Green SE, Francis JJ, et al. Evaluation of the fidelity of an interactive face-to-face educational intervention to improve general practitioner management of back pain. BMJ Open 2015;5:e007886.

12. Perepletchikova F, Hilt LM, Chereji E, et al. Barriers to implementing treatment integrity procedures: survey of treatment outcome researchers. J Consult Clin Psychol 2009;77:212-8.

13. Wang B, Stanton B, Deveaux L, et al. Factors influencing implementation dose and fidelity thereof and related student outcomes of an evidence-based national HIV prevention program. Implement Sci 2015;10:44.

14. Masterson-Algar P, Burton CR, Rycroft-Malone J, et al. Towards a programme theory for fidelity in the evaluation of complex interventions. J Eval Clin Pract 2014;20:445-52.

15. Langford $\mathrm{R}$, Bonell $\mathrm{C}$, Jones $\mathrm{H}$, et al. Obesity prevention and the Health promoting Schools framework: essential components and barriers to success. Int J Behav Nutr Phys Act 2015;12:15.

16. Mowbray CT, Holter MC, Teague GB, et al. Fidelity Criteria: development, Measurement, and validation. Am J Eval 2003;24:315-40.

17. Poltawski L, Norris M, Dean S. Intervention fidelity: developing an experience-based model for rehabilitation research. $J$ Rehabil Med 2014:46:609-15.

18. Bellg AJ, Borrelli B, Resnick B, et al. Enhancing treatment fidelity in health behavior change studies: best practices and recommendations from the NIH Behavior Change Consortium. Health Psychol 2004;23:443-51.

19. Lorencatto F, West R, Christopherson C, et al. Assessing fidelity of delivery of smoking cessation behavioural support in practice. Implement Sci 2013;8:8.

20. Borrelli B. The Assessment, monitoring, and enhancement of treatment Fidelity In Public Health clinical trials. J Public Health Dent 2011;71(s1):S52-S63.

21. Huijg JM, Dusseldorp E, Gebhardt WA, et al. Factors associated with physical therapists' implementation of physical activity interventions in the Netherlands. Phys Ther 2015;95:539-57.

22. McKenna JW, Flower A, Ciullo S. Measuring Fidelity to improve intervention effectiveness. Interv Sch Clin 2014;50:15-21.

23. Resnicow K, Davis M, Smith M, et al. How best to measure implementation of school health curricula: a comparison of three measures. Health Educ Res 1998;13:239-50.

24. Breitenstein SM, Gross D, Garvey CA, et al. Implementation fidelity in community-based interventions. Res Nurs Health 2010;33:n/a-73.

25. Hurley DA, Hall AM, Currie-Murphy L, et al. Theory-driven groupbased complex intervention to support self-management of osteoarthritis and low back pain in primary care physiotherapy: protocol for a cluster randomised controlled feasibility trial (SOLAS). BMJ Open 2016;6:e010728. 
26. Hurley DA, Murphy LC, Hayes D, et al. Using intervention mapping to develop a theory-driven, group-based complex intervention to support self-management of osteoarthritis and low back pain (SOLAS). Implementation Science 2016;11:1-29.

27. Toomey E, Matthews J, Guerin S, et al. Development of a Feasible Implementation Fidelity Protocol within a Complex Physical TherapyLed Self-Management intervention. Phys Ther 2016:96:1287-98.

28. Creswell JW, Plano Clark VL. Designing and Conducting Mixed methods Research. 2 ed. Thousand Oaks, CA: SAGE Publications 2011.

29. Barbour RS. The case for combining qualitative and quantitative approaches in health services research. J Health Serv Res Policy 1999;4:39-43.

30. Ivankova NV, Creswell JW, Stick SL. Using Mixed-Methods Sequential Explanatory Design: from Theory to practice. Field methods 2006;18:3-20.

31. Hardeman W, Michie S, Fanshawe T, et al. Fidelity of delivery of a physical activity intervention: predictors and consequences. Psychol Health 2008;23:11-24.

32. Breitenstein SM, Fogg L, Garvey C, et al. Measuring implementation fidelity in a community-based parenting intervention. Nurs Res 2010;59:158-65.

33. Perepletchikova F, Kazdin AE. Treatment integrity and Therapeutic Change: issues and Research Recommendations. Clinical Psychology: Science and Practice 2005;12:365-83.

34. Garbacz LL, Brown DM, Spee GA, et al. Establishing treatment fidelity in evidence-based parent training programs for externalizing disorders in children and adolescents. Clin Child Fam Psychol Rev 2014;17:230-47.

35. Braun V, Clarke V. Using thematic analysis in psychology. Qual Res Psychol 2006;3:77-101.

36. Guerin S, Hennessy E. Pupils' definitions of bullying. European Journal of Psychology of Education 2002;17:249-61.

37. Wendler MC. Triangulation using a meta-matrix. J Adv Nurs 2001;35:521-5

38. Farmer T, Robinson K, Elliott SJ, et al. Developing and implementing a triangulation protocol for qualitative health research. Qual Health Res 2006;16:377-94.
39. Fleiss J. Reliability of Measurement. Fleiss J, ed. The design and analysis of clinical experiments. New York: wiley, 1986:1-32.

40. Carroll K, Nich C, Rounsaville B. Utility of Therapist session checklists to Monitor delivery of Coping skills treatment for Cocaine Abusers. Psychotherapy Research 1998;8:307-20.

41. Borrelli B. The Assessment, monitoring, and enhancement of treatment Fidelity In Public Health clinical trials. J Public Health Dent 2011;71:S52-S63.

42. Leventhal H, Friedman MA. Does establishing fidelity of treatment help in understanding treatment efficacy? comment on Bellg et al. (2004). Health Psychol 2004;23:452-6.

43. Pincus T, Anwar S, McCracken LM, et al. Delivering an Optimised Behavioural intervention (OBI) to people with Low back pain with High Psychological risk; results and lessons learnt from a feasibility randomised Controlled trial of Contextual Cognitive Behavioural therapy (CCBT) vs. physiotherapy. BMC Musculoskelet Disord 2015;16:147.

44. Palinkas LA, Aarons GA, Horwitz S, et al. Mixed method designs in implementation research. Adm Policy Ment Health 2011;38:44-53.

45. Taylor CA, Shaw RL, Dale J, et al. Enhancing delivery of health behaviour change interventions in primary care: a meta-synthesis of views and experiences of primary care nurses. Patient Educ Couns 2011;85:315-22.

46. Hill LG, Maucione K, Hood BK. A focused approach to assessing program fidelity. Prev Sci 2007;8:25-34.

47. Dane AV, Schneider BH. Program integrity in primary and early secondary prevention: are implementation effects out of control? Clin Psychol Rev 1998;18:23-45.

48. Castro FG, Barrera M, Martinez CR. The cultural adaptation of prevention interventions: resolving tensions between fidelity and fit. Prev Sci 2004;5:41-5.

49. Durlak JA, DuPre EP. Implementation matters: a review of research on the influence of implementation on program outcomes and the factors affecting implementation. Am J Community Psychol 2008;41(3-4):327-50.

50. Oakley A, Strange V, Bonell C, et al. Process evaluation in randomised controlled trials of complex interventions. $B M J$ 2006;332:413-6. 